\title{
A New Risk Model Predicting Overall Survival Using Preoperative Nutritional and Inflammation Status (NIS) in Patients After Curative Resection of Colorectal Cancer
}

\section{Tamuro Hayama ( $\square$ tamuro@med.teikyo-u.ac.jp )}

Teikyo University School of Medicine

Tsuyoshi Ozawa

Teikyo University School of Medicine

Mitsuo Tsukamoto

Teikyo University School of Medicine

Yoshihisa Fukushima

Teikyo University School of Medicine

Ryu Shimada

Teikyo University School of Medicine

Keijiro Nozawa

Teikyo University School of Medicine

Keiji Matsuda

Teikyo University School of Medicine

Shoichi Fujii

Yokohama general hospital

Takeo Fukagawa

Teikyo University School of Medicine

Yojiro Hashiguchi

Teikyo University School of Medicine

\section{Research Article}

Keywords: colon cancer, NLR, CAR, PNI, GPS

Posted Date: May 7th, 2021

DOI: https://doi.org/10.21203/rs.3.rs-467144/v1

License: (1) (1) This work is licensed under a Creative Commons Attribution 4.0 International License. Read Full License 
Page 2/16 


\section{Abstract}

It has been shown that nutritional status correlates with survival in patients with various kinds of cancers. Besides, cancer causes inflammation which has been suggested to stimulate cancer progression. Therefore, inflammation status also has shown to reflect prognosis of cancers. In this study, we evaluated several kinds of nutritional and inflammation parameters in preoperative blood samples and constructed new risk model predicting a survival in patients with CRC (colorectal cancers). We retrospectively examined 286 patients with stage I-III CRC who had undergone curative resection in Teikyo University Hospital between 2013 to 2017. The association between overall survival (OS) and preoperative body mass index, albumin (Alb), cholesterol (Chol), and lymphocyte count, white blood cell count (WBC), neutrophil count (Neu), platelet count (PIt), C-reactive protein (CRP) were examined using Kaplan-Meier curve and log rank test. and eventually Alb, Chol, Neu, Plt, and CRP were shown to correlate with OS. Alb, Chol, Neu, Plt, and CRP were shown to correlate with OS. We constructed a new risk model (NIS: nutrition inflammation status) using these factors, and compared its usefulness with known models such as CRPalbumin ratio (CAR), Glasgow prognostic score (GPS), prognostic nutritional index (PNI), and neutrophil lymphocyte ratio (NLR). NIS prepared using nutritional indicators and inflammatory findings was useful as a new model for predicting overall survival in patients undergoing curative resection for CRC, compared with known models.

\section{Introduction}

In recent years, as typified by the term Precision medicine, identification of recurrence risk factors according to individual conditions and formulation of recurrence risk classification, which exceeds the TNM classification, have been sought. Recent studies have reported that preoperative nutritional status and internal inflammatory findings as patient factors correlate with postoperative recurrence risk, and that diets that cause colorectal inflammation increase the risk of CRC (colorectal cancer). It suggests a relationship between inflammation and colorectal cancer (1-3). We clinicians need a simple prognostic tool for CRC. Simple prognostic tools have been developed using inflammatory markers and nutrition index. Control of nutritional status (CONUT), which consists of albumin (ALB), total lymphocyte count (TLC), and total cholesterol serum levels, GPS (Glasgow prognosis score), and PNI (prognostic nutritional index) is a widely established predictor for cancer patients (4-6). However, its accuracy is still insufficient and there is no classification ability that exceeds the TNM classification. This study, we aimed to develop a tool using nutritional indicators and inflammatory markers that indicate excellent OS.

\section{Patients And Methods}

\section{Patient selection}

Stage I-III CRC diagnosed based on the 8th edition (27) of the United States Joint Commission on Cancer (AJCC) staging system and undergoing radical resection at Teikyo University Hospital in Japan between 2013and 2017. We enrolled 286 consecutive patients. This study has been approved by Teikyo University 
comittee (Registration Number; 19-153). A written informed consent was obtained from all participants, the reporting of our research is in accordance with the STROBE guidelines (28).

\section{GPS score}

The GPS score is as follows: score 2; hypoalbuminemia $(<3.5 \mathrm{~g} / \mathrm{dL})$ and the elevated C-reactive protein (CRP) $(>1.0 \mathrm{mg} / \mathrm{dL})$, score 1 ; hypoalbuminemia $(<3.5 \mathrm{~g} / \mathrm{dL})$ or $\mathrm{CRP}>1.0 \mathrm{mg} / \mathrm{dL}$, score 0 ; albumin concentrations $\geq 3.5 \mathrm{~g} / \mathrm{dL}$ and $\mathrm{CRP} \leq 1.0 \mathrm{mg} / \mathrm{dL}(29)$.

\section{PNI}

The PNI calculated using serum albumin and the peripheral lymphocyte count is a simple and useful score for predicting the prognosis for various cancer patients (30), where PNI = serum albumin level $(\mathrm{g} / \mathrm{dL})+5 \times$ total lymphocyte count (31). Onodera reported that this index provided an accurate, quantitative estimate of operative risk (32). In general, resection and anastomosis of the gastrointestinal tract can be safely practiced when the index is $>45$. The same procedure may be dangerous when the index is between 45 and 40 . When the $\mathrm{PNI}$ is $<40$, this surgery may be contraindicated (32).

\section{Indices of general condition: NLR, CAR}

NLR is the neutrophil / lymphocyte ratio. CAR is the C-reactive protein / albumin ratio. NLR and CAR were obtained by taking blood within 1-2 week preoperatively.

\section{Follow-up}

Surgical resection was defined as curative when there was no evidence of tumor recurrence and the distant metastases were histologically and macroscopically complete. Patients were followed up every 3 months for the first 3 years, every 6 months for the next 2 years. At each follow-up, all patients underwent physical examination and measurements of CEA (serum carcinoembryonic antigen) and CA19-9 (carbohydrate antigen 19-9). They also underwent colonoscopy 1-2 years after surgery (rectal cancer was every year after surgery). Thoracoabdominal computed tomography scans were usually taken every 6 months. Recurrence was defined as the appearance of a radiological, clinical, and / or pathological diagnosis of cancer cells that were local or distant from their original location.

\section{Statistical analysis}

Overall survival (OS) were calculated from the date of the patient underwent surgery to that of death, using the Kaplan-Meier method. A Cox regression analysis was performed to identify factors that are significantly associated with OS. Probability (p)-values $\leq 0.05$ were considered significant. All statistical analyses were performed using JMP 15 software (SAS, Cary, NC, USA).

\section{Determination of cut-off values}

The continuous values are based on the Receiver operating characteristics (ROC) curve, and Youden-index is the Cut-off value. The comparison was made by dividing into two groups, a high-value group and a low- 
value group. For each value, a ROC curve was created by plotting the sensitivity and specificity of each result under investigation. The score closest to the point with both maximum sensitivity and specificity was selected as the cutoff value, and the largest number of tumors were correctly classified for clinical outcome.

The interpretation of an index of probability of concordance (C-index) between predicted probability and actual outcome was used so as to evaluate the predictive ability and discrimination of the model. The value of the C-index should fall between 0.5 and 1.0 , with 0.5 indicating random chance and 1.0 indicating a perfect discriminative ability ( $R$ version 4.0 .3 software).

The AIC (Akaike information criterion) is a popular method for comparing the adequacy of multiple, possibly nonnested models (33). A statistic that evaluates the predictability of a statistical model using the difference between the observed value and the theoretical value. The smaller the value, the better the fit (34).

\section{Results}

\section{Patient characteristics}

The study included a total of 286 patients. The median age was 67.5 (range, 28-93) years; 166 (58.0\%) patients were male and 120 (42.0\%) were female. T factor (the depth of tumor invasion) was 97 (33.9\%) for T1 or T2, and $189(66.1 \%)$ for T3 or T4. There were 106 (37.0\%) cases with lymph node metastasis (N factor $\mathbb{\nabla}$ ) and $180(62.9 \%)$ cases without lymph node metastasis ( $N$ factor -$)$, GPS was $0,1,2(196,59,31)$ (Table.1).

\section{Determination of cut-off values}

The ROC curve analysis results in 3yer-OS indicated that the most appropriate cutoff value for WBC was 6000 . All patients were categorized into the high WBC group (WBC $\geq 6000 ; n=140,49.0 \%$ ) and a low WBC group (WBC $₫ 6000 ; n=146,51.0 \%$ ). Neu were classified into a high Neu group (Neu $\geq 4307 ; n=102,35.6 \%$ ) and a low Neu group (Neu囚4307; n=184, 64.3\%). Plt were classified into a high Plt group (PIt $\geq 194.6$; $n=43,15.0 \%$ ) and a low Plt group (Plt $194.6 ; n=243,85.0 \%$ ). Alb were classified into a high Alb group (Alb $\geq 3.9 ; n=161,56.2 \%$ ) and a low Alb group (Alb囚3.9; n=125, 43.7\%). Lym were classified into a high Lym group (lym $\geq 1606 ; n=96,33.6 \%$ ) and a low Lym group (Lymヌ1606; $n=190,66.4 \%$ ). Chol were classified into a high Chol group (Chol $\geq 119 ; n=272,95.1 \%$ ) and a low Chol group (Chol $₫ 119 ; n=14,4.9 \%)$. NLR were classified into a high NLR group (NLR $\geq 2.24 ; n=200,70.0 \%$ ) and a low NLR group (NLR 2.24; $n=86$, $30.0 \%$ ). CAR were classified into a high CAR group (CAR $\geq 0.025 ; n=154,53.8 \%$ ) and a low CAR group( CAR囚0.025; $n=132,46.2 \%$ )(Table.1).

\section{Relationship between nutritional findings and overall survival}

BMI (body mass index), lym (lymphocyte count), Alb (albumin), and Chol (cholesterol ) were used as nutritional indicators. The Kaplan-Meier curve of the OS in each item is shown in Fig.1. BMI and 
lymphocyte count were not significant factors. On the other hand, the low Alb group had a significantly worse overall survival rate than the high Alb group $\quad(p<0.0002)$. Similarly, Chol had significantly worse overall survival in the low Chol group than in the high Chol group $(p<0.0001)$ (Fig.1).

\section{Relationship between Inflammatory findings and overall survival}

WBC (White blood cell count), Neu (neutrophil count), Plt (platelets), and CRP (C-reactive protein) were used as inflammatory findings. The Kaplan-Meier curve of the OS in each item is shown in Fig.2. WBC were not a significant factor in the relationship for overall survival $(p=0.234)$. On the other hand, in the high Neu group, high Plt group, and high CRP group, the overall survival rate is significantly worse than that of the low Neu group, low Plt group, and low CRP group, respectively. It was $(p=0.017,0.006$, 0.0003) (Fig. 2).

Relationship between a combination model of known nutritional and inflammatory findings (NLR, CAR, PNI)】tumor marker and overall survival

As known models, NLR and CAR were used as inflammation indicators, and PNI and GPS were used as nutritional indicators. NLR was not a significant factor in known models and overall survival $(p=0.051)$. CAR, PNI, and GPS were the indicators significantly associated with overall survival $(p=0.0001, p$ $<0.0001, p=0.006$ ) (Fig. 3). CEA and CA19-9 were used as tumor markers, but CA19-9 was not a significant factor $(p=0.427)$. On the other hand, the overall survival rate was significantly worse in the high CEA group than in the low CEA group $(p=0.002)$.

\section{Stratification using patient factors}

Univariate analysis was performed using the COX proportional hazard model for the factors of BMI, Alb, Lym, Chol, WBC, Neu, PIt, and CRP in the OS. We decided to create a new risk model using five factors that were significant: Alb, Chol, Neu, Plt, and CRP (Table 3). The risk model was calculated by adding up the above-mentioned number of risk factors. The factors high group is positive. NIS counted that one positive was 1, 2 was 2, 3 was 3,4 was 4 , and 5 was 5 . Kaplan-Meier survival curve was drawn by dividing into positive numbers (Fig. S1). As a result, 3 or more were defined as the high value group and 2 or less as the low value group. We named this new risk model NIS (nutrition inflammation status).

Kaplan-Meier Curve of NIS

Survival analyses were performed between low NIS group and high NIS group according to cutoff value of NIS. Statistically significant differences between the two groups were revealed by Kaplan-Meier curves on 3-year OS ( $\mathrm{P}<0.0001)$, indicating a potential prognostic value of NIS. The 3-year OS were $97.1 \%$ for the low NIS group, 77.3\% for the high NIS group, respectively. A survival curve comparison of 3-year OS between the low NIS and high NIS groups showed a significantly poorer prognosis in the high NIS group (Fig.4).

Evaluation of NIS using C-index and AIC compared with T factor and N factor, CAR, PNI, GPS, CEA 
$\mathrm{T}$ factor and $\mathrm{N}$ factor were used as oncological factors. Univariate and multivariate analysis was performed using the Cox proportional hazard model to determine the effects of $\mathrm{T}$ factor and $\mathrm{N}$ factor, CAR, PNI, GPS, CEA, and NIS on overall survival. As a result of multivariate analysis, only three significant factors, $\mathrm{N}$ factor $\mathrm{PNI}$ and NIS, were extracted $(\mathrm{HR}=2.9,95 \%$ confidence interval $(\mathrm{Cl}): 1.3-6.5, \mathrm{HR}=4.8$, 95\%Cl:1.50-15.5, HR $=4.4,95 \% \mathrm{Cl}$ : 1.5-12.9, respectively). As shown in Table2, C-indexes for the score (N factor, PNI, NIS) to predict OS were $0.636,0.726$ and 0.747 , respectively. The AIC values of each index for OS were 277.85 for $\mathrm{N}$ factor, 265.32 for the $\mathrm{PNI}, 257.34$ for the NIS. According to this comparison, NIS was the best goodness-of-fit, followed by $\mathrm{N}$ factor and GPS. NIS was the best model to reflect the prognosis after colorectal cancer surgery compared to CAR, PNI and GPS (table.2).

\section{Discussion}

Currently, there are various indicators that clinically indicate the nutritional status of patients and the findings of inflammation in the body. There are serum protein level, serum albumin level, serum prealbumin level, serum transferrin level, and lymphocyte count as markers indicating nutritional status, and the Glassgow prognostic score (GPS) and prognostic nutritional index (PNI) that combine these markers are reported. Has been done. In addition, white blood cell count, its fraction, platelet count, Creacted protein (CRP) and neutrophil lymphocyte ratio (NLR) combined with them, CRP-Albumin ratio (CAR), etc. are indicators of inflammatory findings in the body. It has been reported. I It has been reported that BMI (14), NLR (15), CAR (16), PNI (17), and GPS (18) are factors related to the prognosis of patients undergoing curative resection for CRC. We conducted a study with the aim of constructing a new selection model of high-risk recurrence group that combines tumor factors, preoperative nutritional status as these patient factors, and findings of internal inflammation.

The items used in the new risk model were five factors that were significantly associated with OS: alb, chol, Neu, plate, and CRP. Each of the five factors has been reported to be associated with OS. Albumin is produced by hepatocytes, and reflects the nutritional status and amount of skeletal muscle, and is decreased by inflammatory mediators (7) (8). Hypoalbuminemia often may reflect the presence of progressive disease and poor performance status caused by tumor cachexia. The role of pretreatment serum albumin as a prognostic factor was demonstrated by many studies. Cholesterol is widely distributed not only in the blood but also throughout the body such as the brain, internal organs, and muscles, and is a material for bile that helps digestion and absorption of cell membranes, sex hormones, corticosteroids, and fat (9). Total cholesterol levels are known as an indicator of a patient's reserve calories (10). Other studies have reported that low serum cholesterol levels have a poor prognosis for various cancer patients (11-13). The relationship between low total blood cholesterol and cancer mortality has been reported in many previous studies. It is thought that this is mainly because the total cholesterol level decreases with the development of cancer, especially with the progression of colorectal cancer and advanced cancer (14). Therefore, hypocholesterolemia is not considered to be the cause of cancer, but it is thought to be caused by cancer (13). In this study, it was a poor prognosis factor in the group with a large number of neutrophils. 
The mechanism underlying the association between a large number of neutrophils and worse outcomes has not yet been completely clarified. However, it could be attributed to the association of neutrophils with inflammation. Neutrophils can inhibit the immune system, thereby eliminating the cytolytic activity of immune cells (15) (16). Therefor both tumor and host cells including neutrophils can simultaneously produce chemokines and cytokines, thus contributing to tumor progression (17).

Rao X et al reported that an elevated platelet count is a negative predictor of survival in both primary CRC and resectable colorectal liver metastases(18). Many studies have shown that increased platelet count promotes the growth, infiltration, and metastasis of cancer $(19,20)$. Platelets can secrete a variety of growth factors and angiogenesis regulatory proteins (IL-6, vascular endothelial growth factor, plateletderived growth factor, platelet factor 4) to promote tumor formation and its metastasis (21) (22).

Allin et al reported that patients with invasive breast cancer and diagnostic CRP levels $>3 \mathrm{mg} / \mathrm{L}$ had a 1.7fold increased risk of death from breast cancer compared to patients with diagnostic CRP levels $<1 \mathrm{mg} / \mathrm{L}$ (30). Biological mechanisms associated with elevated CRP lebels and poor cancer prognosis may explain inflammation in the tumor microenvironment. Inflammation of the tumor microenvironment induces DNA damage, promotes angiogenesis, and promotes tumor spread and distant metastasis. The result is an attractive tumorigenesis promoting environment for tumor growth (23-25).

Currently, TNM staging is most used to predict survival outcomes and treatment choices. However, because TNM staging was done postoperatively, survival cannot be predicted preoperatively. Also, no further treatment strategy can be determined. In addition, the TNM stage can only reflect the biological behavior of the tumor. The prognosis of cancer was associated with nutritional status not only with the clinicopathological features of the tumor, but also with the inflammatory response of the host (1) (26). NIS is based on peripheral neutrophils, platelet count, CRP, blood albumin levels, cholesterol levels, reflects the state of the tumor microenvironment and the preoperative host inflammatory response and nutritional status. Our findings show that preoperative NIS has stronger prognostic discrimination capabilities compared to NLR, CAR, GPS, and PNI. Therefore, the use of a combination of parameters that reflects the host of both the nutritional status and the systemic inflammatory status may be important for accurately predicting survival outcomes in CRC patients.

This study has its limitations. First, this study was retrospective in design and included patients from a single institution. Second, this study was small sample size. Third, there are no consensus regarding the nutrition and inflammatory markers cut-off value. We selected nutrition and inflammatory markers cut-off value by performing a ROC analysis. Our current findings require further review and validation in more CRC patients due to the smaller number of cases. Fourth, the results of this study do not apply to stage IV patients, as they included stage I-III CRC patients who underwent curative surgery.

\section{Conclusion}

NIS prepared using nutritional indicators and inflammatory findings was useful as a new model for predicting overall survival in patients undergoing curative resection for $\mathrm{CRC}$, compared with known 
models. It was suggested that it could be used clinically in the future.

\section{Abbreviations}

AIC Akaike's information criterion

AJCC American Joint Committee on Cancer

CA19-9 carbohydrate antigen 19-9

CEA carcinoembryonic antigen

C-INDEX index of probability of concordance

CONUT Controlling Nutritional Status

CRC colorectal cancer

CRP C-reactive protein

GPS Glasgow Prognostic Score

NIS nutrition inflammation status

PNI Prognostic Nutritional Index

OS overall survival

\section{Declarations}

The authors declare that they have no conflict of interest

Funding: none

Compliance with ethical standards:

The present study was conducted in accord with the Declarations of Helsinki and was approved by the Ethics Committee of the Teikyo University (approval date, 23 August 2016, registration number; 16-032).

Acknowledgements: I am grateful to 20th Fujii Tomoko academic encouragement award and ACRO Research Grants of Teikyo University for collaboration on the early stages of this work.

\section{References}

1. Hayama T, Ozawa T, Okada Y, Tsukamoto M, Fukushima Y, Shimada R, et al. The pretreatment Controlling Nutritional Status (CONUT) score is an independent prognostic factor in patients 
undergoing resection for colorectal cancer. Sci Rep. 2020;10(1):13239.

2. Chen JH, Zhai ET, Yuan YJ, Wu KM, Xu JB, Peng JJ, et al. Systemic immune-inflammation index for predicting prognosis of colorectal cancer. World journal of gastroenterology. 2017;23(34):6261-72.

3. Zhang J, Zhang HY, Li J, Shao XY, Zhang CX. The elevated NLR, PLR and PLT may predict the prognosis of patients with colorectal cancer: a systematic review and meta-analysis. Oncotarget. 2017;8(40):68837-46.

4. Minami S, Ogata Y, Ihara S, Yamamoto S, Komuta K. Pretreatment Glasgow prognostic score and prognostic nutritional index predict overall survival of patients with advanced small cell lung cancer. Lung Cancer (Auckland, NZ). 2017;8:249-57.

5. Miyake M, Morizawa Y, Hori S, Marugami N, lida K, Ohnishi K, et al. Integrative Assessment of Pretreatment Inflammation-, Nutrition-, and Muscle-Based Prognostic Markers in Patients with Muscle-Invasive Bladder Cancer Undergoing Radical Cystectomy. Oncology. 2017;93(4):259-69.

6. Watanabe J, Otani S, Sakamoto T, Arai Y, Hanaki T, Amisaki M, et al. Prognostic indicators based on inflammatory and nutritional factors after pancreaticoduodenectomy for pancreatic cancer. Surgery today. 2016;46(11):1258-67.

7. Visser M, Kritchevsky SB, Newman AB, Goodpaster BH, Tylavsky FA, Nevitt MC, et al. Lower serum albumin concentration and change in muscle mass: the Health, Aging and Body Composition Study. The American journal of clinical nutrition. 2005;82(3):531-7.

8. Fuhrman MP. The albumin-nutrition connection: separating myth from fact. Nutrition (Burbank, Los Angeles County, Calif). 2002;18(2):199-200.

9. Kuzu OF, Noory MA, Robertson GP. The Role of Cholesterol in Cancer. Cancer Res. 2016;76(8):206370.

10. Gadgil MD, Anderson CA, Kandula NR, Kanaya AM. Dietary patterns are associated with metabolic risk factors in South Asians living in the United States. J Nutr. 2015;145(6):1211-7.

11. Ko K, Park YH, Lee JW, Ku JH, Kwak C, Kim HH. Influence of nutritional deficiency on prognosis of renal cell carcinoma (RCC). BJU international. 2013;112(6):775-80.

12. Cubiella J, Castells A, Fondevila C, Sans M, Sabater L, Navarro S, et al. Prognostic factors in nonresectable pancreatic adenocarcinoma: a rationale to design therapeutic trials. The American journal of gastroenterology. 1999;94(5):1271-8.

13. Zhou P, Li B, Liu B, Chen T, Xiao J. Prognostic role of serum total cholesterol and high-density lipoprotein cholesterol in cancer survivors: A systematic review and meta-analysis. Clinica chimica acta; international journal of clinical chemistry. 2018;477:94-104.

14. de Ulíbarri Pérez JI, Fernández G, Rodríguez Salvanés F, Díaz López AM. Nutritional screening; control of clinical undernutrition with analytical parameters. Nutr Hosp. 2014;29(4):797-811.

15. el-Hag A, Clark RA. Immunosuppression by activated human neutrophils. Dependence on the myeloperoxidase system. Journal of immunology (Baltimore, Md : 1950). 1987;139(7):2406-13. 
16. Petrie HT, Klassen LW, Kay HD. Inhibition of human cytotoxic T lymphocyte activity in vitro by autologous peripheral blood granulocytes. Journal of immunology (Baltimore, Md : 1950). 1985;134(1):230-4.

17. Dell'Aquila E, Cremolini C, Zeppola T, Lonardi S, Bergamo F, Masi G, et al. Prognostic and predictive role of neutrophil/lymphocytes ratio in metastatic colorectal cancer: a retrospective analysis of the TRIBE study by GONO. Annals of oncology : official journal of the European Society for Medical Oncology. 2018;29(4):924-30.

18. Rao XD, Zhang H, Xu ZS, Cheng H, Shen W, Wang XP. Poor prognostic role of the pretreatment platelet counts in colorectal cancer: A meta-analysis. Medicine. 2018;97(23):e10831.

19. Riedl J, Pabinger I, Ay C. Platelets in cancer and thrombosis. Hamostaseologie. 2014;34(1):54-62.

20. Radziwon-Balicka A, Santos-Martinez MJ, Corbalan JJ, O'Sullivan S, Treumann A, Gilmer JF, et al. Mechanisms of platelet-stimulated colon cancer invasion: role of clusterin and thrombospondin 1 in regulation of the P38MAPK-MMP-9 pathway. Carcinogenesis. 2014;35(2):324-32.

21. Peterson JE, Zurakowski D, Italiano JE, Jr., Michel LV, Connors S, Oenick M, et al. VEGF, PF4 and PDGF are elevated in platelets of colorectal cancer patients. Angiogenesis. 2012;15(2):265-73.

22. Pilatova K, Greplova K, Demlova R, Bencsikova B, Klement GL, Zdrazilova-Dubska L. Role of platelet chemokines, PF-4 and CTAP-III, in cancer biology. Journal of hematology \& oncology. 2013;6:42.

23. Coussens LM, Werb Z. Inflammation and cancer. Nature. 2002;420(6917):860-7.

24. Vakkila J, Lotze MT. Inflammation and necrosis promote tumour growth. Nature reviews Immunology. 2004;4(8):641-8.

25. Mantovani A, Allavena P, Sica A, Balkwill F. Cancer-related inflammation. Nature. 2008;454(7203):436-44.

26. Hayama T, Hashiguchi Y, Okada Y, Ono K, Nemoto K, Shimada R, et al. Significance of the 7th postoperative day neutrophil-to-lymphocyte ratio in colorectal cancer. International journal of colorectal disease. 2020;35(1):119-24.

27. Weiser MR. AJCC 8th Edition: Colorectal Cancer. Annals of surgical oncology. 2018;25(6):1454-5.

28. von Elm E, Altman DG, Egger M, Pocock SJ, Gøtzsche PC, Vandenbroucke JP. The Strengthening the Reporting of Observational Studies in Epidemiology (STROBE) Statement: guidelines for reporting observational studies. International journal of surgery (London, England). 2014;12(12):1495-9.

29. McMillan DC, Elahi MM, Sattar N, Angerson WJ, Johnstone J, McArdle CS. Measurement of the systemic inflammatory response predicts cancer-specific and non-cancer survival in patients with cancer. Nutr Cancer. 2001;41(1-2):64-9.

30. Tominaga T, Nonaka T, Hisanaga M, Fukuda A, Tanoue Y, Yoshimoto T, et al. Prognostic value of the preoperative prognostic nutritional index in oldest-old patients with colorectal cancer. Surgery today. 2020;50(5):449-59.

31. Nozoe T, Kohno M, Iguchi T, Mori E, Maeda T, Matsukuma A, et al. The prognostic nutritional index can be a prognostic indicator in colorectal carcinoma. Surgery today. 2012;42(6):532-5. 
32. Onodera T, Goseki N, Kosaki G. [Prognostic nutritional index in gastrointestinal surgery of malnourished cancer patients]. Nihon Geka Gakkai zasshi. 1984;85(9):1001-5.

33. Vrieze SI. Model selection and psychological theory: a discussion of the differences between the Akaike information criterion (AIC) and the Bayesian information criterion (BIC). Psychological methods. 2012;17(2):228-43.

34. H. A. A new look at the statistical model identification. IEEE Transactions on Automatic Control. 1974;19:716-23.

\section{Tables}

Table 1. Clinicopathological features of the stage I- I I colorectal cancer patients who underwent curative tumor resection

\begin{tabular}{|c|c|c|}
\hline Clinicopathological feature & $\mathrm{N}=286(\%)$ & Cut off value \\
\hline Gender $\$ male/Female $\rrbracket$ & $166(58.0) / 120(42.0)$ & - \\
\hline Age (range, median) & $28-93,67.5$ & - \\
\hline BMI (kg/m2)(low / high) & $68(76.2) / 218(23.8)$ & 25.2 \\
\hline 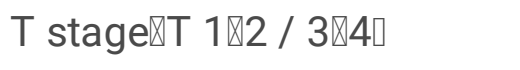 & $97(33.9) / 189(66.1)$ & - \\
\hline 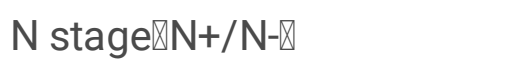 & $106(37.0) / 180$ (62.9) & - \\
\hline CEA (ng/mL)(low / high) & $137(47.9) / 149(52.1)$ & 3.5 \\
\hline CA19-9 (U/mL)(low / high) & $252(88.1) / 34(11.9)$ & 50.2 \\
\hline Alb (g/dL)(low / high) & $125(43.7)$ / $161(56.2)$ & 3.9 \\
\hline Lym (mm3)(low / high) & $190(66.4) / 96(33.6)$ & 1606 \\
\hline Chol (mg/dl)(low / high) & $14(4.9) / 272(95.1)$ & 119 \\
\hline WBC (/pl)(low / high) & $146(51.0) / 140(49.0)$ & 6000 \\
\hline Neu (mm3)(low / high) & $184(64.3) / 102(35.6)$ & 4307 \\
\hline Plt (mm3)(low / high) & $243(85.0)$ / 43 (15.0) & 19.5 \\
\hline CRP (mg/dL)(low / high) & $168(58.7) / 118(41.3)$ & 0.12 \\
\hline NLR (low / high) & $86(30.0) / 200(70.0)$ & 2.24 \\
\hline CAR (low / high) & $132(46.6) / 154(53.8)$ & 0.025 \\
\hline PNI (low / high) & $154(53.8) / 132(46.1)$ & 46.23 \\
\hline mGPS $(0 / 1 / 2)$ & 196 (68.5), 59 (20.6), $31(10.8)$ & - \\
\hline
\end{tabular}


Table 2. Univariate and multivariate analysis results for the 3-year OS using Cox proportional hazard model, C-index and AIC.

\begin{tabular}{|lllllllll|}
\hline Factors & \multicolumn{6}{ll}{ Univariate analysis } & \multicolumn{7}{c|}{ Multivariate analysis } \\
& HR & $95 \% \mathrm{Cl}$ & $\mathrm{p}$ & $\mathrm{HR}$ & $95 \% \mathrm{Cl}$ & $\mathrm{p}$ & $\mathrm{C}-$-index & AIC \\
\hline TNM & 13.8 & $1.9-102$ & 0.01 & & & & & \\
CAR & 5.5 & $2.1-14.4$ & 0.001 & & & & & \\
PNI & 7.5 & $2.6-21.6$ & 0.0002 & 4.8 & $1.5-15.5$ & 0.009 & 0.726 & 265.32 \\
GPS & 4.2 & $1.6-10.9$ & 0.011 & & & & & \\
NIS & 8.3 & $3.8-18.1$ & $<0.0001$ & 4.4 & $1.5-12.9$ & 0.008 & 0.747 & 257.34 \\
\hline
\end{tabular}

Table 3. Univariate analysis of nutritional and inflammatory findings using Cox proportional hazard model in 3-year OS

\begin{tabular}{|llll|}
\hline Factors & \multicolumn{3}{l|}{ Univariate analysis } \\
& HR & $95 \% \mathrm{Cl}$ & $\mathrm{p}$ \\
\hline BMI & 2.0 & $0.70-5.9$ & 0.161 \\
\hline Alb & 4.2 & $1.8-9.9$ & 0.0004 \\
\hline Lym & 2.23 & $0.8-5.9$ & 0.105 \\
\hline Chol & 5.8 & $2.2-15.6$ & 0.003 \\
\hline WBC & 1.59 & $0.7-3.4$ & 0.234 \\
\hline Neu & 2.45 & $1.1-5.2$ & 0.02 \\
\hline Plt & 2.9 & $1.3-6.5$ & 0.015 \\
\hline CRP & 4.33 & $1.8-10.3$ & 0.0003 \\
\hline
\end{tabular}

Figures 
Fig.1 A
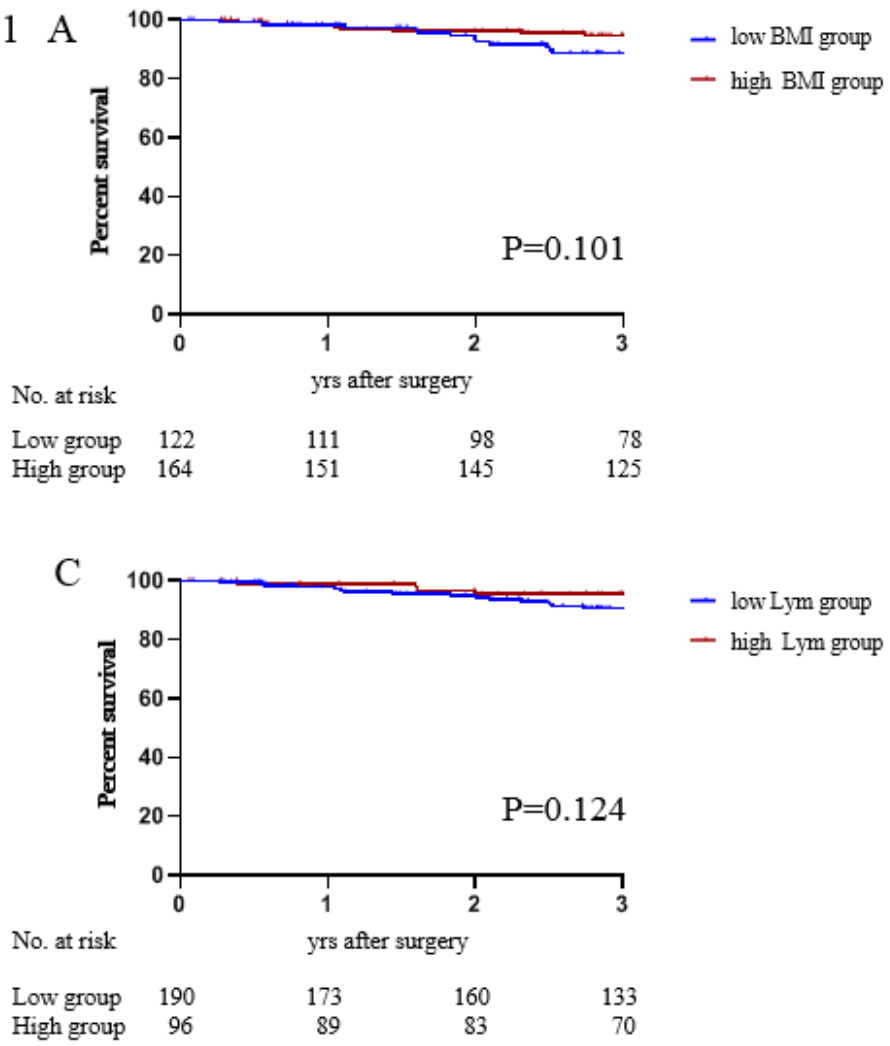

B

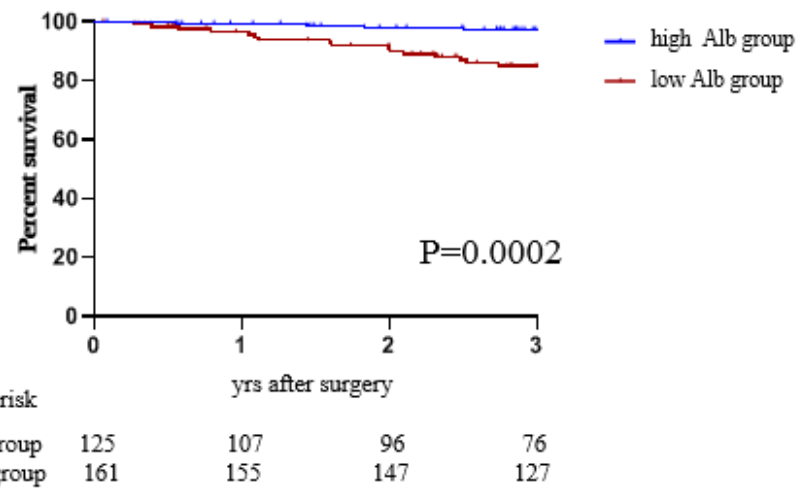

D

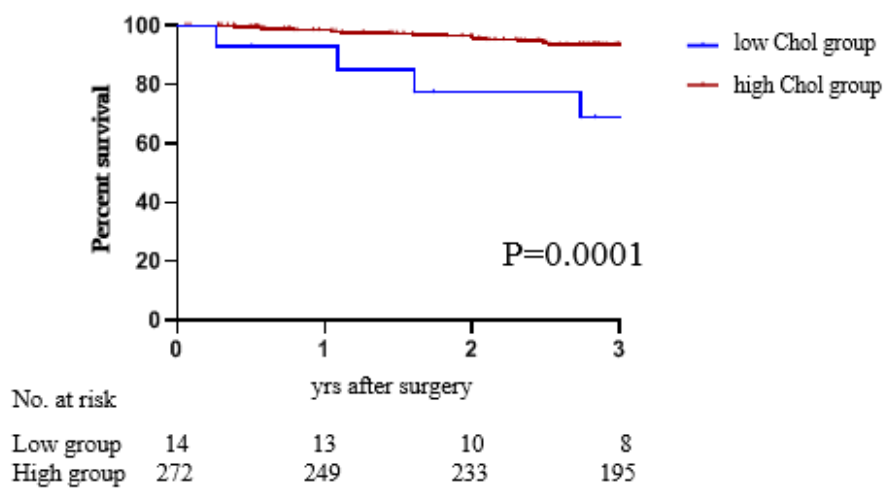

\section{Figure 1}

Kaplan-Meier curves of 3 years OS based on the nutriton index. (A) BMI, (B) Alb, (C) Lym, and (D) Chol.

Fig. 2
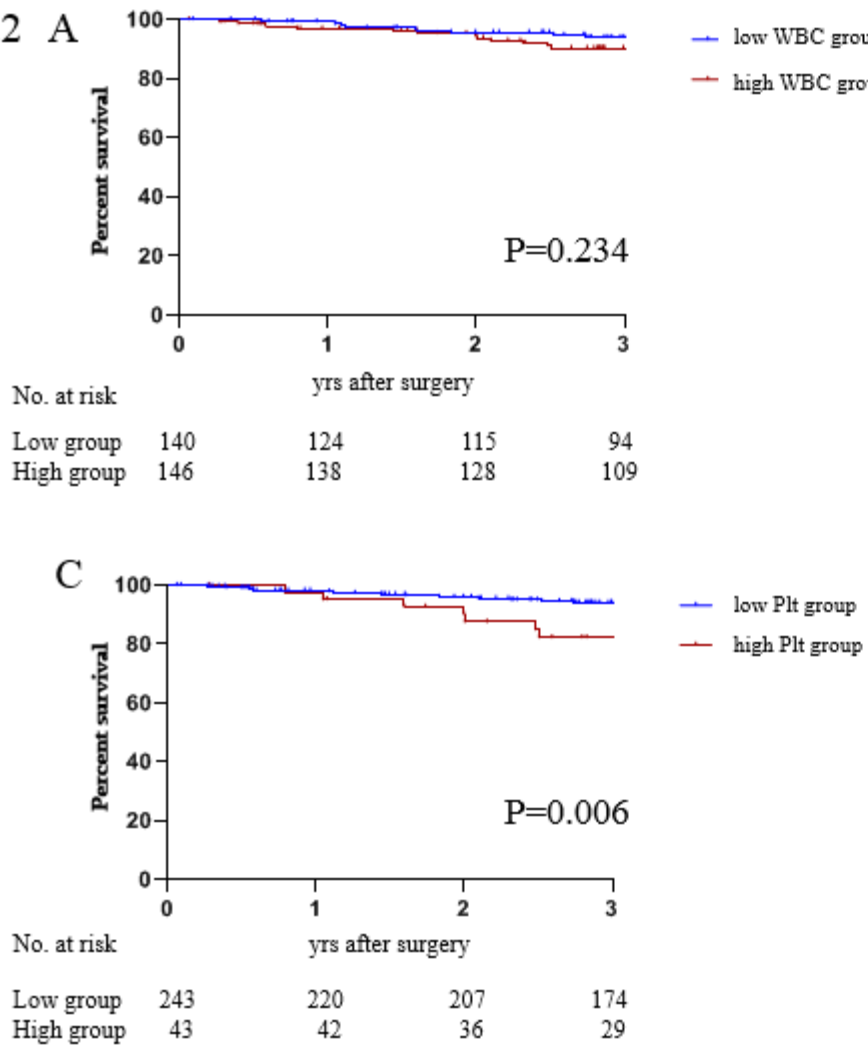

B

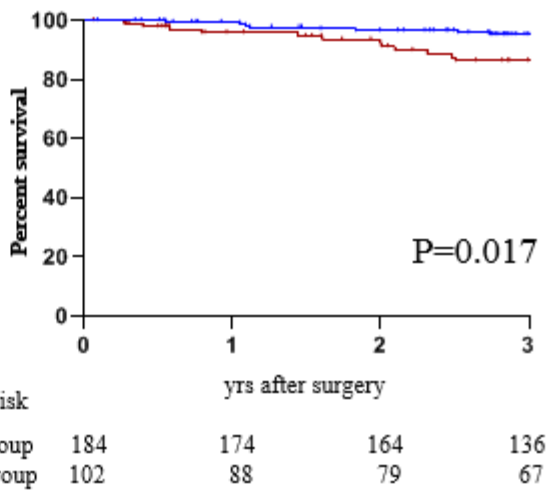

D

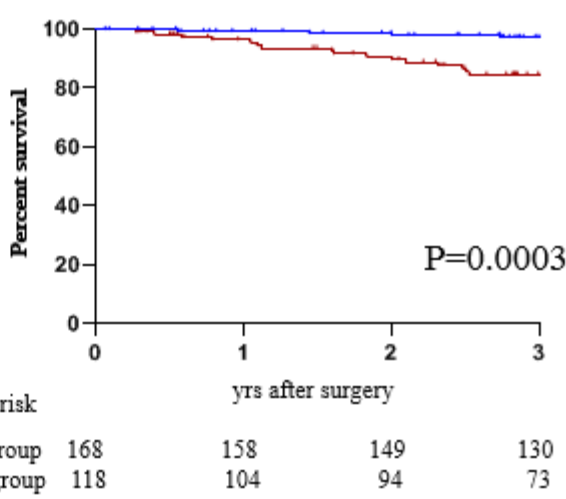

- low Neu group

- high Neu group

Figure 2 
Kaplan-Meier curves of 3 years OS based on the inflammatory makers. (A) WBC, (B) Neu, (C) Plt, and (D) CRP.

Fig.3 A
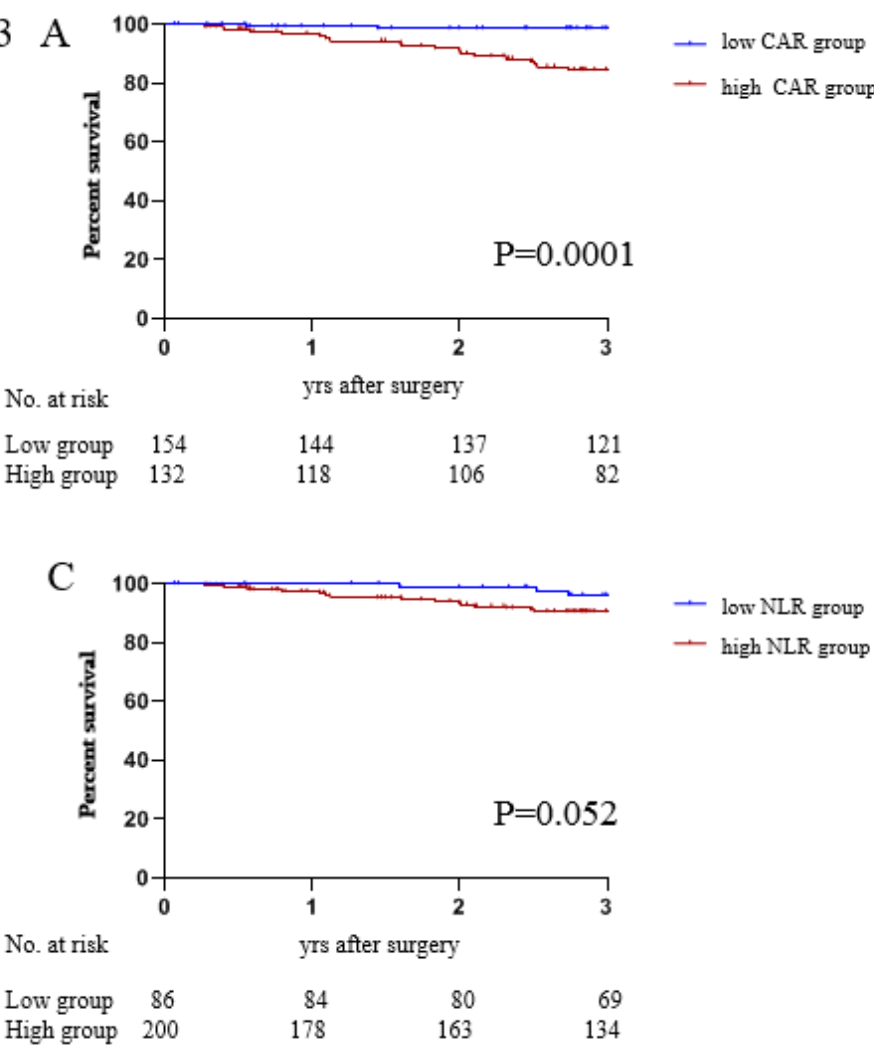

B

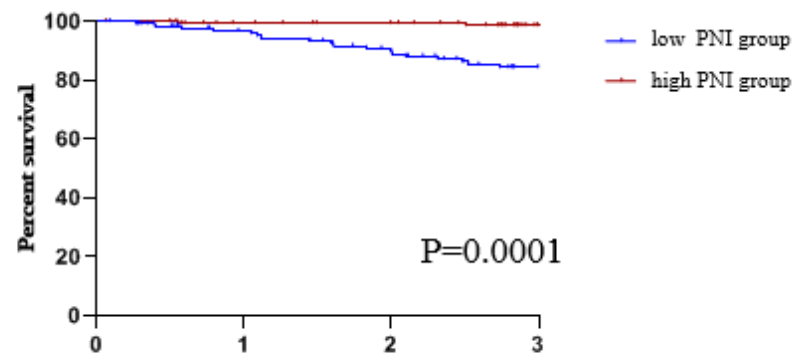

No. at risk

$\begin{array}{lllll}\text { Low group } & 132 & 116 & 102 & 84\end{array}$

$\mathrm{D}$

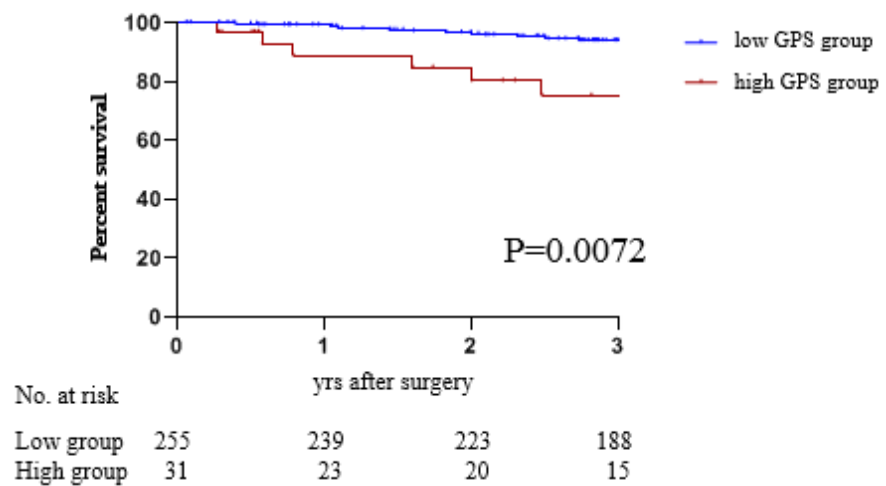

\section{Figure 3}

Kaplan-Meier curves of 3 years OS based on the known nutritional and inflammatory findings. (A) CAR, (B) PNI, (C) NLR,(D) GPS.

Fig.4

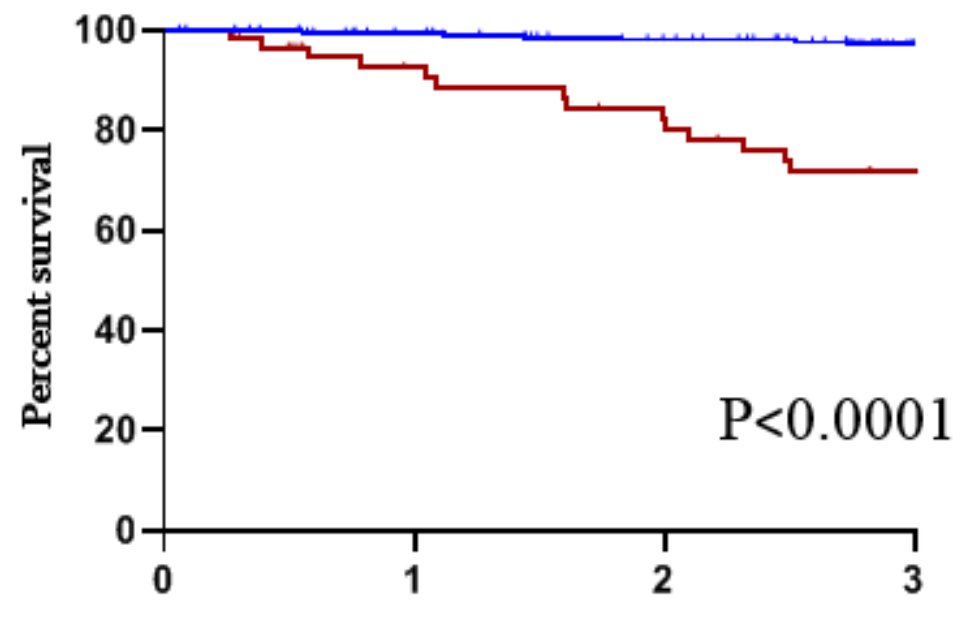

No. at risk

yrs after surgery

$\begin{array}{lrrrr}\text { Low group } & 228 & 215 & 203 & 171 \\ \text { High group } & 58 & 47 & 40 & 32\end{array}$


Figure 4

Kaplan-Meier curves of 3 years OS based on the NIS (low group and high group).

\section{Supplementary Files}

This is a list of supplementary files associated with this preprint. Click to download.

- Fig.S1.pptx 